OPEN ACCESS

Edited by:

Baolei Jia

Chung-Ang University, South Korea

Reviewed by:

Susana Merino,

University of Barcelona, Spain

Guang Zhao,

Qingdao Institute of Bioenergy and Bioprocess Technology (CAS),

China

Raquel Regina Bonelli, Federal University of Rio de Janeiro,

Brazil

*Correspondence: Boyang Cao

boyangcao@nankai.edu.cn

†These authors have contributed equally to this work

Specialty section: This article was submitted to Evolutionary and Genomic Microbiology,

a section of the journal Frontiers in Microbiology

Received: 12 December 2018

Accepted: 25 March 2019

Published: 10 April 2019

Citation:

Xi D, Wang X, Ning K, LiU Q, Jing F, Guo $X$ and Cao B (2019)

O-Antigen Gene Clusters

of Plesiomonas shigelloides Serogroups and Its Application in Development of a Molecular

Serotyping Scheme.

Front. Microbiol. 10:741. doi: 10.3389/fmicb.2019.00741

\section{O-Antigen Gene Clusters of Plesiomonas shigelloides Serogroups and Its Application in Development of a Molecular Serotyping Scheme}

\author{
Daoyi Xi1,2,3,4t, Xiaochen Wang ${ }^{1,2,3,4 \dagger}$, Kexin Ning 1,2,3,4, Qian Liu'1,2,3,4, Fuyi Jing ${ }^{1,2,3,4}$, \\ Xi Guo ${ }^{1,2,3,4}$ and Boyang Cao ${ }^{1,2,3,4 *}$ \\ ${ }^{1}$ Key Laboratory of Molecular Microbiology and Technology of the Ministry of Education, TEDA College, Nankai University, \\ Tianjin, China, ${ }^{2}$ TEDA Institute of Biological Sciences and Biotechnology, Nankai University, Tianjin, China, ${ }^{3}$ Tianjin Research \\ Center for Functional Genomics and Biochips, TEDA College, Nankai University, Tianjin, China, ${ }^{4}$ Tianjin Key Laboratory \\ of Microbial Functional Genomics, TEDA College, Nankai University, Tianjin, China
}

Plesiomonas shigelloides is a Gram-negative, flagellated, rod-shaped, ubiquitous, and facultative anaerobic bacterium. It has been isolated from various sources, such as freshwater, surface water, and many wild and domestic animals. P. shigelloides is associated with diarrheal diseases of acute secretory gastroenteritis, an invasive shigellosis-like disease, and a cholera-like illness in humans. At present, 102 somatic antigens and 51 flagellar antigens of $P$. shigelloides have been recognized; however, very little is known about variations of $\mathrm{O}$-antigens among $P$. shigelloides species. In this study, 12 O-antigen gene clusters of $P$. shigelloides, O2H1a1c (G5877), O10H41 (G5892), O12H35 (G5890), O23H1a1c (G5263), O25H3 (G5879), O26H1a1c (G5889), O32H37 (G5880), O33H38 (G5881), O34H34 (G5882), O66H3 (G5270), O75H34 (G5885), and O76H39 (G5886), were sequenced and analyzed. The genes that control O-antigen synthesis are present as chromosomal gene clusters that maps between rep and aqpZ, and most of the synthesis and translocation of OPS (O-specific polysaccharide) belongs to Wzx/Wzy pathway with the exception of 012,025 , and O66, which use the ATPbinding cassette $(A B C)$ transporter pathway. Phylogenetic analysis of wzx and wzy show that the wzx and wzy genes are specific to individual O-antigens and can be used as targets in molecular typing. Based on the sequence data, an O-antigen specific suspension array that detects 12 distinct OPS' has been developed. This is the first report to catalog the genetic features of $P$. shigelloides $O$-antigen variations and develop a suspension array for the molecular typing. The method has several advantages over traditional bacteriophage and serum agglutination methods and lays the foundation for easier identification and detection of additional O-antigen in the future.

Keywords: Plesiomonas shigelloides, O-antigen gene clusters, serogroups, PCR, luminex-based array 


\section{INTRODUCTION}

Plesiomonas shigelloides was first isolated from the feces of a patient for whom no clinical history was available. It was reported to be a motile organism with the major antigen of Shigella sonnei phase I, and originally named Paracolon C27 by Ferguson and Henderson (1947), (Hu et al., 2013). Biochemical, morphological, and taxonomical features of the organism were collected by many investigators and the organism was reclassified into the family Enterobacteriaceae, being the only oxidasepositive member of this family (Martinez-Murcia et al., 1992; Huys and Swings, 1999; Garrity et al., 2001). P. shigelloides, the only species of the genus, is a Gram-negative, flagellated, rodshaped, ubiquitous, and facultative anaerobic bacterium, which has been later isolated from various sources, such as freshwater, surface water, and many wild and domestic animals (Wong and Pang, 2000). P. shigelloides is associated with diarrheal diseases in humans (Brenden et al., 1988), including acute secretory gastroenteritis (Mandal et al., 1982), an invasive shigellosislike disease (McNeeley et al., 1984), and a cholera-like illness (Tsukamoto et al., 1978). Traveler diarrhea affects millions of tourists each year. $P$. shigelloides has been ranked third in traveler's diarrhea in Japan (Schubert and Holz-Bremer, 1999), and third as a cause of diarrhea among a number of military units in China (Bai et al., 2004), as well as among civilians in Hong Kong (Chan et al., 2003).

The O-antigen is a component of lipopolysaccharides (LPS) present in the outer membrane of Gram-negative bacteria and comprised of repeated oligosaccharide units (O-units, OPS). The OPS is the major contribution of the antigenic variability of the cell surface and is subject to intense selection by the host immune system and other environmental factors, such as bacteriophages, which may account for the existence of diverse O-antigen forms within a species (Reeves et al., 1996). The O-antigen gene clusters consist of genes that belong to three main classes: nucleotide sugar biosynthesis pathway genes, glycosyltransferase (GT) genes, and $\mathrm{O}$-unit processing genes. Three distinct pathways participated in the synthesis and translocation of OPS: the Wzx/Wzy pathway, the ATP-binding cassette (ABC) transporter pathway (Hu et al., 2013), and the synthase pathway. O-antigen serotyping, targeting the $w z x$ and $w z y$ genes, is able to detect distinct O-antigen forms, including E. coli, Shigella, Salmonella, Vibrio parahaemolyticus, Acinetobacter, Hafnia, and Proteus (Li et al., 2009, 2010; Guo et al., 2013; Hu et al., 2013; Chen et al., 2015; Duan et al., 2016; Yu et al., 2017). Our previous studies employed $w z x$ and $w z y$ for serotyping E. coli and Shigella by microarray (Li et al., 2006), wzx and $w z y$ for serotyping Pseudomonas aeruginosa (Li et al., 2018) and wzm and wzt for serotyping 15 Legionella pneumophila serogroups by microarray (Cao et al., 2013). In this report, $w z x, w z y$, and $w z m$ have been screened and used as targets for molecular serotyping of the 12 P. shigelloides strains.

At present, 102 somatic antigens and 51 flagellar antigens of P. shigelloides have been recognized (Aldova and Shimada, 2000). P. shigelloides strain 302-73, isolated in Japan (Shimada and Sakazaki, 1985), is the representative strain for the O1 serogroup, whose complete OPS and the core of LPS has been chemically characterized (Pieretti et al., 2010), and the whole-genome sequence of $P$. shigelloides 302-73 have been obtained in Pique et al. (2013). P. shigelloides O17 is one of the most frequently encountered serovar, which is involved with protection against shigellosis as it carries a LPS in the cell walls that is also common to S. sonnei (Sayeed et al., 1992).

In this study, $12 \mathrm{O}$-antigen gene clusters of $P$. shigelloides, serogroups O2, O10, O12, O23, O25, O26, O32, O33, O34, O66, O75, and O76, were sequenced and analyzed. The genes that control $\mathrm{O}$-antigen synthesis are present as chromosomal gene clusters that maps between rep and $a q p Z$, and most of the synthesis and translocation of OPS occurs within the Wzx/Wzy pathway, with the exception of O12, O25, and O66, which use ABC transporter pathway. Phylogenetic analysis of $w z x$ and $w z y$ show that the $w z x$ and $w z y$ genes are specific to individual $\mathrm{O}$-antigens and can be used as targets in molecular typing.

In summary, the current study: (1) has determined 12 $\mathrm{O}$-antigen gene clusters of $P$. shigelloides serogroups $\mathrm{O} 2, \mathrm{O} 10$, O12, O23, O25, O26, O32, O33, O34, O66, O75, and O76; (2) has analyzed the evolutionary relationship of the $\mathrm{O}$-antigen gene clusters in combination with the published $\mathrm{O}$-antigenspecific gene data of $P$. shigelloides O1; (3) has established a rapid, bead-based suspension array detection technique using the $w z x, w z y$, or $w z m$ genes of 12 different serogroups of $P$. shigelloides as the molecular serotyping markers. The suspension array method has been tested for its specificity and sensitivity and found to be suitable for applications in the fields of clinical research, diagnosis, treatment, epidemiology, and prevention of diseases.

\section{MATERIALS AND METHODS}

\section{Bacterial Strains}

The 12 P. shigelloides O-standard reference strains employed in this study are listed in Table $\mathbf{1 .}$

\section{Genomic DNA Extraction}

All P. shigelloides strains were cultured in Tryptone Soya Broth (TSB) (QingDao ShuiRi Bio-Technologies Co., Ltd., Qingdao, China) at $37^{\circ} \mathrm{C}$ overnight with shaking. Genomic DNA was prepared by Bacteria Gen DNA Kit (CWBiotech, Beijing, China).

\section{Library Preparation for Illumina Sequencing}

A library for Illumina Paired-End sequencing was prepared from $5 \mu \mathrm{g}$ DNA using a Paired-End DNA Sample Prep Kit (Pe102-1001, Illumina Inc., Cambridge, United Kingdom). DNA was fragmented by nebulization for $6 \mathrm{~min}$ at a pressure of 32 psi. For end-repair and phosphorylation, sheared DNA was purified using the QIAquick Nucleotide Removal Kit (Qiagen, Crawley, United Kingdom). The end-repaired DNA was A-tailed and adaptors were ligated according to the manufacturer's instructions. 
TABLE 1 | Bacterial strains used in this study.

\begin{tabular}{|c|c|c|c|c|}
\hline Bacterium & Lab number & Original number & Original source & Total number \\
\hline Plesiomonas shigelloides $\mathrm{O} 2 \mathrm{H} 1 \mathrm{a} 1 \mathrm{c}$ & G5877 & CNCTC Aer 33/89 & CNCTC & 1 \\
\hline Plesiomonas shigelloides O10h41 & G5892 & CNCTC Aer 58/89 & CNCTC & 1 \\
\hline Plesiomonas shigelloides O12h35 & G5890 & CNCTC Aer 43/89 & CNCTC & 1 \\
\hline Plesiomonas shigelloides $\mathrm{O} 23 \mathrm{H} 1 \mathrm{a} 1 \mathrm{c}$ & G5263 & A1 & Italy & 1 \\
\hline Plesiomonas shigelloides $\mathrm{O} 25 \mathrm{H} 3$ & G5879 & CNCTC Aer 37/89 & CNCTC & 1 \\
\hline Plesiomonas shigelloides $\mathrm{O} 26 \mathrm{H} 1 \mathrm{a} 1 \mathrm{c}$ & G5889 & CNCTC Aer 36/89 & CNCTC & 1 \\
\hline Plesiomonas shigelloides $\mathrm{O} 32 \mathrm{H} 37$ & G5880 & CNCTC Aer 61/89 & CNCTC & 1 \\
\hline Plesiomonas shigelloides $\mathrm{O} 33 \mathrm{H} 38$ & G5881 & CNCTC Aer 62/89 & CNCTC & 1 \\
\hline Plesiomonas shigelloides $034 \mathrm{H} 34$ & G5882 & CNCTC Aer 35/89 & CNCTC & 1 \\
\hline Plesiomonas shigelloides $\mathrm{O} 66 \mathrm{H} 3$ & G5270 & $\mathrm{H} 3$ & Italy & 1 \\
\hline Plesiomonas shigelloides $075 \mathrm{H} 34$ & G5885 & CNCTC Aer 3/65 & CNCTC & 1 \\
\hline Plesiomonas shigelloides $\mathrm{O} 76 \mathrm{H} 39$ & G5886 & CNCTC Aer 55/89 & CNCTC & 1 \\
\hline
\end{tabular}

CNCTC, Czechia National Collection of Type Cultures, the Czechia. Department of Environmental Sciences, Naples, Italy.

\section{Draft Genome Sequencing, Assembling, and Annotation}

As reported previously ( $\mathrm{Hu}$ et al., 2013), the whole-genome sequencing was performed using an Illumina/Solexa genome analyzer IIx (Illumina, San Diego, CA, United States). De novo assembly was performed using SOAP de novo (Xie et al., 2014). Gene prediction and annotation were carried out using the NCBI Prokaryotic Genome Annotation Pipeline (Tatusova et al., 2016). The Staden package and the Artemis program were used for sequence assembly and gene annotation, respectively. The BlockMaker program was used to search for conserved motifs. BLAST and PSIBLAST (Altschul et al., 1997) were used to search sequence databases, including the GenBank database and the Pfam protein motif database (Bateman et al., 2002), to identify potential gene functions. The program TMHMM 2.0 (Krogh et al., 2001) was used to identify potential transmembrane segments. Sequence alignments and comparisons were performed using the ClustalW program (Thompson et al., 1994). For sugar pathway genes, a BLAST search against the UniProt/SwissProt database was used to confirm the allocation of the genes by pathway.

\section{Phylogenetic Analysis}

A phylogenetic tree was constructed using the neighbor-joining method and plotted by the Molecular Evolutionary Genetics Analysis (MEGA) 6.0 software package. Bootstrap analysis was carried out based on 1,000 replicates.

\section{Target Genes and Primer Design}

The target genes for primer and probe design were $w z x$ genes of $P$. shigelloides $\mathrm{O} 2, \mathrm{O} 10$, and $\mathrm{O} 26$, wzy genes of $P$. shigelloides $\mathrm{O} 23$, O32, O33, O34, O75, and O76, and $w z m$ genes of $P$. shigelloides O12, O25, and O66 (Table 2). Primers were designed by Primer Premier (Yang et al., 2018). The forward primer was biotinylated at the $5^{\prime}$-end to allow binding to the reporter dye streptavidin-R-phycoerythrin for detection on a Bio-Plex platform. All the primer sequences used for the multiplex PCR are listed in Table 2.

\section{Multiplex PCR and Labeling of the Target Genes}

Multiplex PCR was carried out in two groups of six serogroups each; Group A consists of $P$. shigelloides O12, O25, O26, O33, O75, and O76; Group B consists of P. shigelloides O2, O10, O23, O32, O34, and O66. The multiplex PCR amplification was performed with $50 \mu \mathrm{L}$ of a reaction mixture that consisted of $100 \mathrm{ng}$ of DNA, $25 \mu \mathrm{L}$ of HotStar Taq Master Mix (Qiagen), $0.25 \mu \mathrm{L}$ of the forward primer, and $1.0 \mu \mathrm{L}$ of the reverse one, with an exception of $\mathrm{O} 25$ with double the amount of both primers. Reaction parameters were: $95^{\circ} \mathrm{C}$ for $5 \mathrm{~min} ; 35$ cycles of $94^{\circ} \mathrm{C}$ for $30 \mathrm{~s}, 54^{\circ} \mathrm{C}$ for $45 \mathrm{~s}$, and $72^{\circ} \mathrm{C}$ for $50 \mathrm{~s}$; and a final extension at $72^{\circ} \mathrm{C}$ for $5 \mathrm{~min}$. An aliquot of $2 \mu \mathrm{L}$ of PCR product was run on an agarose gel to examine the amplified DNA. After amplification, the samples were stored at $-20^{\circ} \mathrm{C}$ until used.

\section{Probe Design and Bead Coupling}

All species-specific probes were based on the sequence data from the lab data. Multiple-sequence alignments were carried out using BioEdit version 7.0 software. These species-specific probes were newly designed (Table 2). Probes were synthesized with a $5^{\prime}$-end amino C-12 modification (AuGCT, Beijing, China) and coupled to carboxylated beads (Bio-Rad Laboratories, Hercules, CA, United States) as described by the manufacture's manual. Briefly, a total of $2.5 \times 10^{5}$ carboxylated beads were suspended in $8.5 \mu \mathrm{L}$ of $0.1 \mathrm{M} \mathrm{MES} \mathrm{(pH} \mathrm{4.5)} \mathrm{with} 2 \mu \mathrm{L}$ of $0.1 \mathrm{nmol} / \mu \mathrm{L}$ oligonucleotide probes. A $2.5 \mu \mathrm{L}$ of $10 \mathrm{mg} / \mathrm{mL}$ freshly prepared EDC [1-ethyl-3-(3-dimethylaminopropyl) carbodiimide] was added, and vortexed immediately, then incubated at room temperature in the dark for $30 \mathrm{~min}$. After being washed by $0.02 \%$ Tween 20 and $0.1 \%$ SDS, pellets were centrifuged and resuspended in $20 \mu \mathrm{L}$ of TE ( $\mathrm{pH} 8.0)$, and stored at $4^{\circ} \mathrm{C}$ in the dark until used.

\section{Hybridization and Staining}

A bead mix set was prepared, consisting of 2,500 beads for each of the 10 probes in $1.5 \times$ tetramethylammonium chloride (TMAC) solution (Sigma, St. Louis, MO, United States) that contained 4.5 M TMAC, 0.15\% Sarkosyl, 75 mM Tris-HCl 
TABLE 2 | Primers and probes.

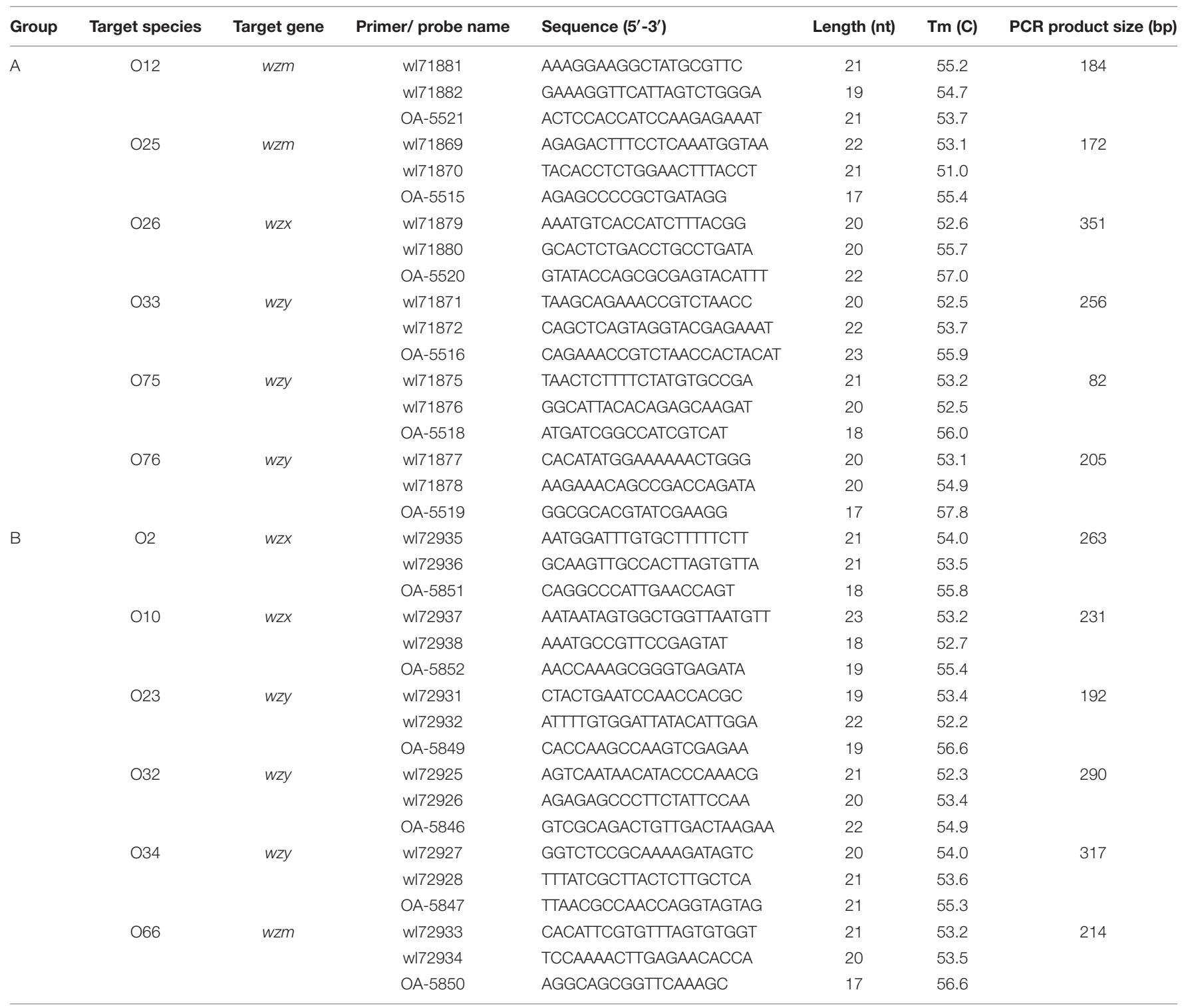

( $\mathrm{pH}$ 8.0), and $6 \mathrm{mM}$ EDTA ( $\mathrm{pH}$ 8.0) solution. A total of $17 \mu \mathrm{L}$ of biotinylated amplicon was added to $33 \mu \mathrm{l}$ of the bead mix. Amplicon and bead mixture were denatured at $95^{\circ} \mathrm{C}$ for $5 \mathrm{~min}$ and allowed to hybridize at $57^{\circ} \mathrm{C}$ for $15 \mathrm{~min}$. The mixture was centrifuged at $8,000 \mathrm{rpm}$ and the supernatant carefully discarded, then the beads were resuspended in $75 \mu \mathrm{L}$ of $1 \times$ TMAC solution containing $10 \mathrm{ng} / \mathrm{mL}$ streptavidin-Rphycoerythrin (Molecular Probes, Eugene, OR, United States) and incubated for $10 \mathrm{~min}$ at $57^{\circ} \mathrm{C}$.

\section{Bead Analysis}

The fluorescence intensity of the beads was analyzed in a BioPlex 100 suspension array system (Bio-Rad Laboratories). The median fluorescence intensities (MFI) were calculated from 100 replicate measurements with a digital signal processor and BioPlex Manager 4.1 software. A positive signal was defined as $\mathrm{MFI}$ is at least $>500$, and the signal/background ratio (S/B ratio $=\mathrm{MFI} /$ Blank) is greater than 3.0.

\section{Sensitivity of the Multiplex PCR Method}

A serial ten-fold dilution of the genomic DNA of $P$. shigelloides $\mathrm{O} 26$ and $\mathrm{O} 75$, ranging from $1.0 \mathrm{fg} / \mathrm{uL}, 10 \mathrm{fg} / \mathrm{uL}, 100 \mathrm{fg} / \mathrm{uL}$, $1.0 \mathrm{pg} / \mathrm{uL}, 10 \mathrm{pg} / \mathrm{uL}, 100 \mathrm{pg} / \mathrm{uL}, 1.0 \mathrm{ng} / \mathrm{uL}, 10 \mathrm{ng} / \mathrm{uL}$, and $100 \mathrm{ng} / \mathrm{uL}$, were used as the template to test the sensitivity of the suspension array assay.

\section{Nucleotide Sequence Accession Number}

The DNA sequences of the O-antigen gene clusters of 12 $\mathrm{O}$-antigen gene clusters of $P$. shigelloides serogroups $\mathrm{O} 2, \mathrm{O} 10$, O12, O23, O25, O26, O32, O33, O34, O66, O75, and $\mathrm{O} 76$ have been deposited in GenBank under the accession numbers MK551179-MK551190, respectively. 


\section{RESULTS}

\section{Allocation and Extraction of OPS Clusters From $P$. shigelloides Genome Sequences}

The O-antigen gene cluster of $12 P$. shigelloides serogroups were obtained and extracted from the type strains of $P$. shigelloides serogroups O2, O10, O12, O23, O25, O26, O32, O33, O34, O66, O75, and O76 (Table 1). The clusters located between rep and $a q p Z$ at the chromosome of these 12 isolates had 22,165, $27,407,16,526,15,506,20,682,29,124,14,430,21,362,25,286$, $21,030,25,342$, and $24,898 \mathrm{bp}$, respectively. Characteristics of the $18,25,11,17,16,30,13,17,20,16,22$, and 18 open reading frames (orfs) of these 12 isolates were identified to each of the serogroups (Figure 1 and Supplementary Tables S1-S12). The functions were assigned based on their similarities to genes of known functions associated with sugar synthesis, sugar transfer, $\mathrm{O}$-antigen processing, and others from available databases (Figures 1, 2).

\section{O-Antigen Cluster of $\boldsymbol{P}$. shigelloides $\mathrm{O2}$}

For the synthesis of UDP-L-FucNAc, FnlABC is reportedly involved in synthesizing $\mathrm{O}$-polysaccharides in several species, e.g., in Salmonella O47, O48, and O61 (Liu et al., 2014). The set of orf12, orf13, and orf14 in the cluster of $P$. shigelloides O2 shares 93\% identity with FnlA of P. shigelloides WP_010862887, 65 and 84\% with FnlBC of E. coli, indicating the existence of a rare sugar, UDP-L-FucNAc. Rhamnose is widely present as a bacterial surface polysaccharide, and its biosynthesis pathway is well known to require four enzymes that are encoded by $r m l A B C D$ in the polysaccharide gene clusters of E. coli, Shigella, and Salmonella (Liu et al., 2008, 2014). For $P$. shigelloides $\mathrm{O} 2$, the set of orf5, orf6, and orf7 of $P$. shigelloides $\mathrm{O} 2$ shows around $90 \%$ identity to RmlBDA in E. coli, indicating the existence of another rare sugar, dTDP-L-Rha. The orf9 shows 39\% identity to Wzx in Escherichia coli and the orf10 shows $40 \%$ identity to Wzy in Pseudomonas mucidolens. The set of $w z x$ and $w z y$ genes suggests the presence of $\mathrm{Wzx} / \mathrm{Wzy}$ pathway related $\mathrm{O}$-antigen processing. There are two GTs encoded by the orf11 and orf15, which share $37 \%$ identity with GT family 2 protein of Pseudomonas sp. NFR02, and 58\% identity with GT/WbuB of Burkholderia territorii.

\section{O-Antigen Cluster of $P$. shigelloides 010}

The set of FnlABC in the cluster of $P$. shigelloides O10 shares 98\% identity with FnlA of $P$. shigelloides, 63\% identity with FnlB of E. coli, and $99 \%$ identity with FnlC of $P$. shigelloides, indicating the existence of the rare sugar, UDP-L-FucNAc. The function of Ugd is to convert UDP-Glc to UDP-GlcA. The orf7 shares more than 94\% identity with Ugd in Shewanella algae. The orf6 shows $79 \%$ identity to Wzx in Shewanella decolorationis and the orf8 shows 32\% identity to Wzy in Marinobacter sp. EN3. The set of $w z x$ and $w z y$ genes suggests the presence of Wzx/Wzy pathway related $\mathrm{O}$-antigen processing. There are three $\mathrm{GT}$ encoded by the orf9, orf10, and orf 12 which share $40 \%$ identity with GT family 2 protein of Prosthecochloris sp. CIB 2401, 35\% identity with GT
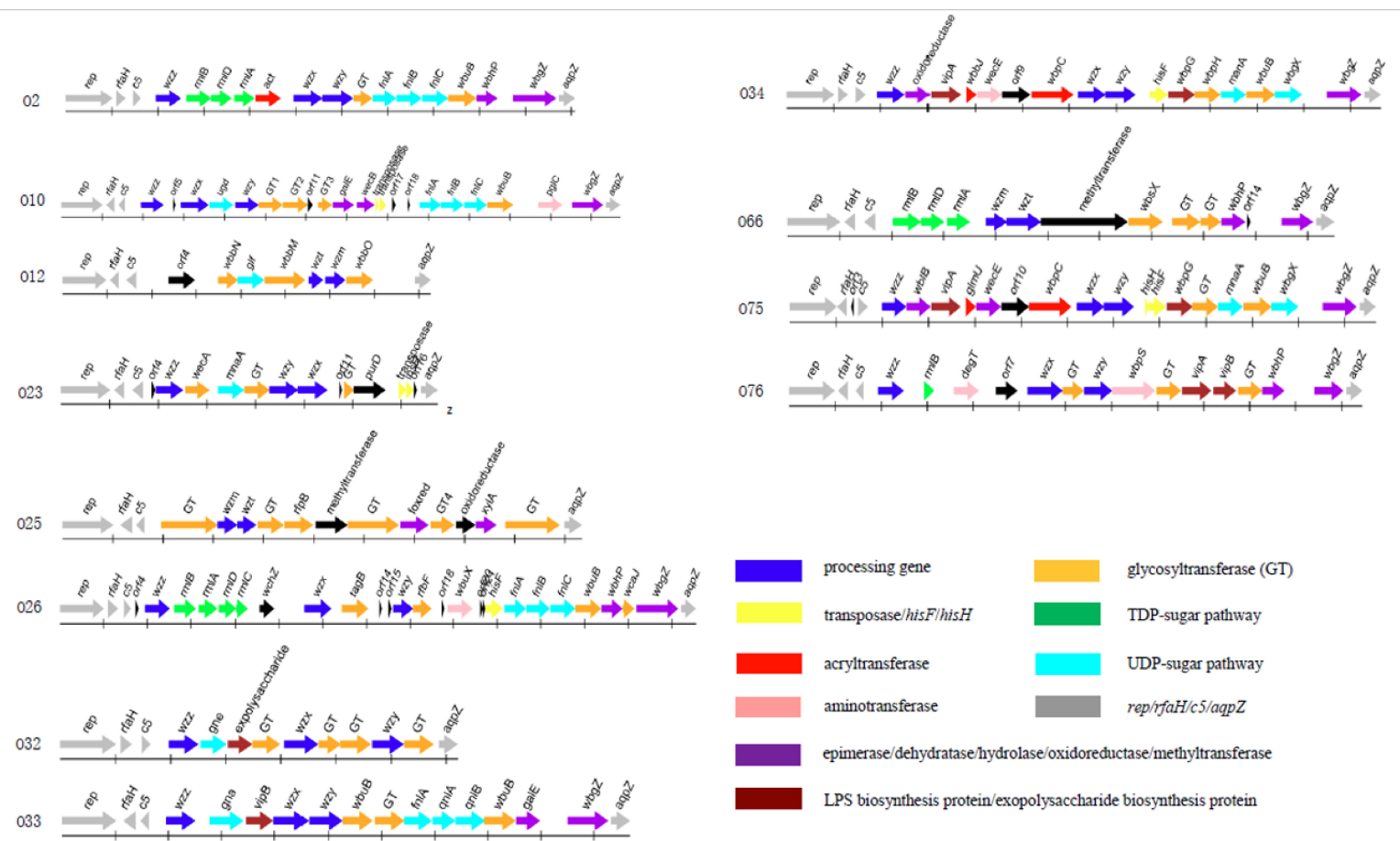

PS biosynthesis protein/exopolysaccharide biosynthesis protein

FIGURE 1 | The O-antigen gene clusters of 12 P. shigelloides serogroups O2, O10, O12, O23, O25, O26, O32, O33, O34, O66, O75, and O76. 

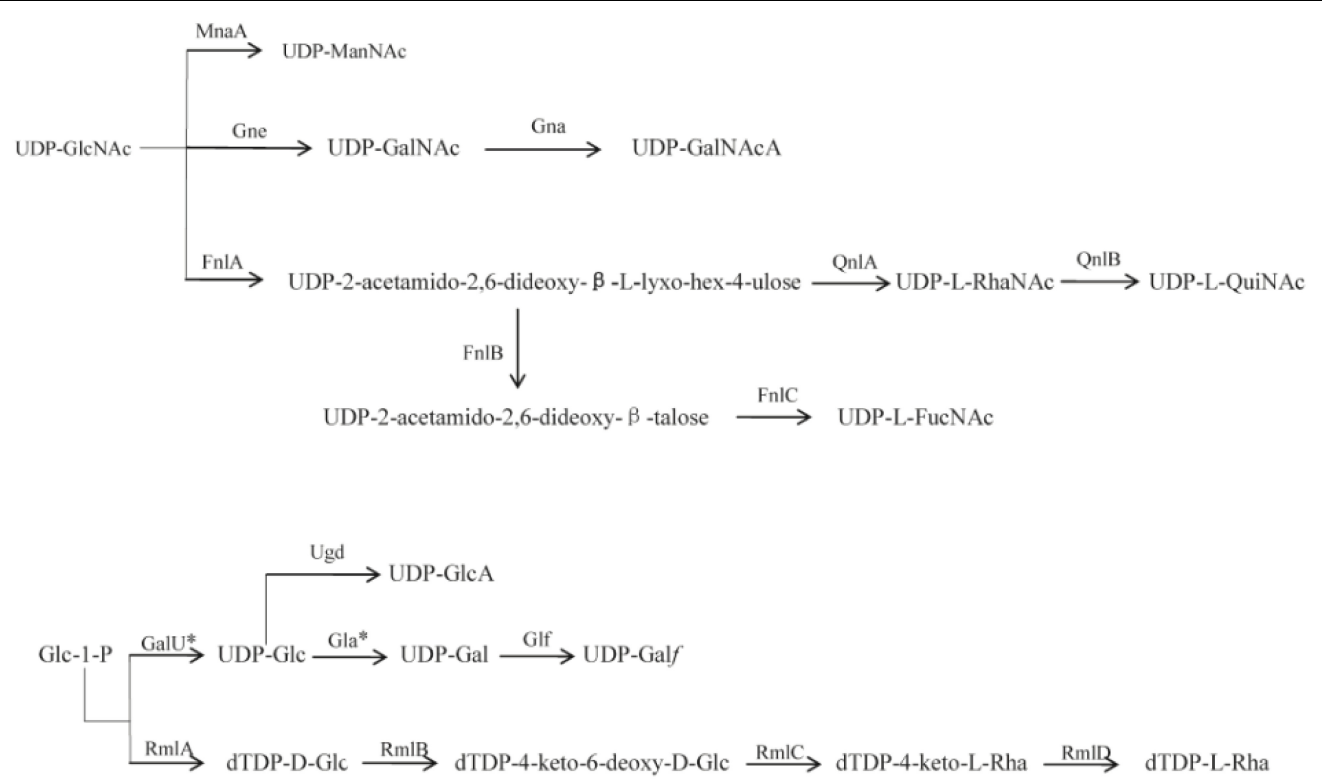

FIGURE 2 | Proposed biosynthesis pathways for sugars in the 12 P. shigelloides serogroups clusters. Putative pathways are denoted by a broken line. MnaA, UDP-N-acetylglucosamine-2-epimerase; Gne, UDP-N-acetylglucosamine-4-epimerase; Gna, UDP-GalNAcA synthetase; FnIA, 4,6-dehydratase, 3- and 5-epimerase; FnIB, reductase; FnIC, C-2 epimerase; QnIA, dTDP-4-dehydrorhamnose reductase; QnIB, C-2 epimerase; GalU*, UTP-glucose-1-phosphate uridylyltransferase; Ugd, UDP-glucose 6-dehydrogenase; Gla*, UDP-galacturonase; Glf, UDP-galactopyranose mutase; RmIA, glucose-1-phosphate thymidylyltransferase; RmIB, dTDP-D-glucose 4,6-dehydratase; RmIC, dTDP-4-keto-6-deoxy-D-glucose 3,5-epimerase; RmID,

dTDP-6-deoxy-L-mannose-dehydrogenase. D-GlcNAc, 2-acetamido-2-deoxy-D-glucose; D-ManNAc, 2-acetamido-2-deoxy-D-mannose; D-GalNAc, 2-acetamido-2-deoxy-D-galactose; D-GalNAcA, 2-Acetamido-2-deoxy-D-galacturonic acid; L-RhaNAc, 2-acetamido-2,6-dideoxy-L-mannose

(N-acetyl-L-rhamnosamine); L-QuiNAc, 2-Acetamido-2-deoxy-L-quinovose (2-acetamido-2,6-dideoxy-L-glucose); L-FucNAc, 2-Acetamido-2-deoxy-L-fucose; D-GlcA, D-glucuronic acid; D-Glc, D-glucose; D-Gla*, D-galactose; D-Galf, D-galactofuranose; L-Rha, L-rhamnose (6-deoxy-L-mannose). *indicates the genes that were found outside the cluster.

family 1 protein of $P$. shigelloides, and $88 \%$ identity with GT of S. algae, respectively.

\section{O-Antigen Cluster of $P$. shigelloides 012}

There is no rare sugar in the cluster of $P$. shigelloides O12. The function of Glf is to convert UDP-Gal to UDP-Galf. The orf6 in the cluster shares $79 \%$ identity with the corresponding gene of Klebsiella pneumoniae. The set of $w z m$ and wzt genes suggests the presence of $\mathrm{ABC}$ transporter pathway related O-antigen processing. There are three GTs encoded by the orf5, orf7, and orf10 which share $50 \%$ identity with GT/WbbN of Serratia liquefaciens, 67\% identity with GT/WbbM of K. pneumoniae subsp. rhinoscleromatis SB3432, and 57\% identity with GT/WbbO of Serratia sp. Leaf50, respectively.

\section{O-Antigen Cluster of $\boldsymbol{P}$. shigelloides $\mathbf{0 2 3}$}

The function of MnaA is to convert UDP-GlcNAc to UDPManNAc. MnaA in P. shigelloides O23 share more than $81 \%$ identity with the one in E. coli, indicating there is a rare sugar UDP-ManNAc in the structure. The orf10 shows $27 \%$ identity to Wzx in E. coli S88 and the orf9 shows $29 \%$ identity to Wzy in Salmonella enterica. The set of $w z x$ and $w z y$ genes suggests the presence of Wzx/Wzy pathway related O-antigen processing. WecA in P. shigelloides O23 shares more than 93\% identity with the corresponding gene of $P$. shigelloides. There is one GT encoded by the orf8 which shares $42 \%$ identity with GT family
4 protein of Vibrio cholerae. There are two transposes encoded by the orf14 and orf15. As Duan et al. (2016) reported in most E. coli and Shigella strains, the first sugar residue in the O-unit is GlcNAc or GalNAc, and the IT (Initial transferase) encoded by wecA, which is responsible for initiating the synthesis of GlcNAcand GalNAc- initiated OPS. Usually, the wecA gene is located outside the cluster and acts as a UDP-GlcNAc:undecaprenyl phosphate GlcNAc-1-phosphate transferase, however, it presents in the $\mathrm{O} 23$ gene cluster.

\section{O-Antigen Cluster of $P$. shigelloides 025}

There is no rare sugar in the cluster of $P$. shigelloides O25. The set of wzm and wzt genes suggests the presence of the $\mathrm{ABC}$ transporter pathway related $\mathrm{O}$-antigen processing. Interestingly, there are six GTs encoded by the orf4, orf7 orf8, orf10, orf12, and orf15 which share 54\% identity with GT family 1 protein of Enterobacteriaceae, 56\% identity with GT family 1 protein of Citrobacter europaeus, 67\% identity with GT/RfbP of C. europaeus, $67 \%$ identity with GT family 1 protein of C. europaeus, $65 \%$ identity with GT family 2 protein of Serratia sp. DD3, and 77\% identity with GT family 2 protein of Duganella sp. Root336D2, respectively.

\section{O-Antigen Cluster of $P$. shigelloides 026}

The orf6 and orf7 of $P$. shigelloides O26 share 89 and 95\% identity with RmlB and RmlA of $V$. cholerae, and the orf8 and orf9 share 
85 and $83 \%$ identity with RmlD and RmlC of Vibrio anguillarum, indicating the presence of a rare sugar, dTDP-L-Rha. The set of orf23, orf24, and orf25 in the cluster shares 93\% identity with FnlA of $P$. shigelloides WP_010862887, 65 and 84\% with FnlBC of $E$. coli, indicating there is a rare sugar UDP-L-FucNAc. The orf 12 shows $36 \%$ identity to Wzx in E. coli and the orf16 shows $36 \%$ identity to Wzy in Flavobacterium sp. GSP27. The presence of $w z x$ and $w z y$ genes suggests the presence of $\mathrm{Wzx} / \mathrm{Wzy}$ pathway related O-antigen processing in O26. Except the two GTs of orf13 and orf28 encoding TagD for teichoic acid biosynthesis and WcaJ for colanic acid biosynthesis. There are other two GTs encoded by the orf 17 and orf 26 which share $38 \%$ identity with GT/RfbF of Shigella flexneri, and $89 \%$ identity with GT/WbuB of $V$. cholerae, respectively.

\section{O-Antigen Cluster of $P$. shigelloides 032}

There is no rare sugar in the cluster of $P$. shigelloides $\mathrm{O} 32$. The orf8 shows $45 \%$ identity to Wzx in E. coli and the orf 11 shows 33\% identity to Wzy in S. enterica. The set of $w z x, w z y$, and $w z z$ genes suggests the presence of $\mathrm{Wzx} / \mathrm{Wzy}$ pathway related O-antigen processing. The orf5 in the cluster of $P$. shigelloides $\mathrm{O} 32$ shares 64\% identity with Gne of Yersinia pseudotuberculosis, indicating there is a sugar UDP- GalNAc. There are four GTs encoded by the orf7, orf9, orf10, and orf12, which share $57 \%$ identity with GT of S. enterica, $57 \%$ identity with GT family 2 protein of Proteus mirabilis, 58\% identity with GT family 4 protein of Edwardsiella tarda, and 55\% identity with GT of Shewanella sp. $\mathrm{SACH}$, respectively.

\section{O-Antigen Cluster of $\boldsymbol{P}$. shigelloides 033}

The orf5 in the cluster of $P$. shigelloides $\mathrm{O} 33$ shares $83 \%$ identity with Gna of $P$. shigelloides, indicating the presence of a sugar, UDP- GalNAcA. The set of orf11, orf12, and orf13 in the cluster of $P$. shigelloides O33 shares more than 93, 69, and 88\% identity with FnlA, QnlA, and QnlB of E. coli, indicating there is a rare sugar, UDP-L-QuiNAc. The orf7 shows $38 \%$ identity to Wzx in E. coli and the orf8 shows $46 \%$ identity to Wzy in Moritella viscosa. There are $w z x$ and $w z y$ genes in the cluster, indicating the presence of $\mathrm{Wzx} / \mathrm{Wzy}$ pathway related $\mathrm{O}$-antigen processing. There are three GTs encoded by orf9, orf10, and orf14 which share $23 \%$ identity with GT/WbuB of Pseudomonas fluorescens, $76 \%$ identity with GT family 1 protein of Aeromonas fluvialis, and $74 \%$ identity with GT/WbuB of Shewanella xiamenensis, respectively.

\section{O-Antigen Cluster of $\boldsymbol{P}$. shigelloides 034}

The orf16 in the cluster of $P$. shigelloides O34 shares more than $89 \%$ identity with MnaA of $V$. cholerae, indicating the presence of UDP-ManNAc. The orf11 shows 36\% identity to Wzx in Janthinobacterium sp. CG23_2 and the orf12 shows 35\% identity to Wzy in Pseudomonas sp. BAY1663. The set of $w z x$, $w z y$, and $w z z$ genes suggest the presence of Wzx/Wzy pathway related $\mathrm{O}$-antigen processing. There are two GTs encoded by the orf15 and orf17 which share $57 \%$ identity with GT/WbpH of Pseudomonas sp. CFII68, and 55\% identity with GT of Photorhabdus luminescens.

\section{O-Antigen Cluster of $\boldsymbol{P}$. shigelloides 066}

The set of orf4, orf5 and orf6 in the cluster of $P$. shigelloides O66 share 91, 94, and 98\% identity with RmlBDA of Aeromonas sp. indicating there is a rare sugar dTDP-L-Rha. The galE is the gene that is responsible for the synthesis of UDP-Gal in the gene cluster. The set of wzm and wzt genes suggest the presence of the $\mathrm{ABC}$ transporter pathway related $\mathrm{O}$-antigen processing. There are three GTs encoded by the orf 10 , orf 11 , and orf 12 which share $63 \%$ identity with GT/WbsX of Nitrosomonas europae, $81 \%$ identity with GT of Aeromonas hydrophila, and 75\% identity with GT family 2 protein of Shigella boydii, respectively.

\section{O-Antigen Cluster of $\boldsymbol{P}$. shigelloides 075}

The MnaA in the cluster of $P$. shigelloides $\mathrm{O} 75$ shares more than $89 \%$ identity with the one of $V$. cholerae, indicating there is a rare sugar, UDP-ManNAc, in the structure. The orf12 shows $42 \%$ identity to Wzx and the orf13 shows 34\% identity to Wzy in Pseudomonas sp. BAY1663. The set of $w z x, w z y$, and $w z z$ genes suggests the presence of Wzx/Wzy pathway related O-antigen processing. There are two GTs encoded by the orf17 and orf19, which share $87 \%$ identity with GT of Vibrio ordalii, and $55 \%$ identity of GT/WbuB of $P$. luminescens.

\section{O-Antigen Cluster of $\boldsymbol{P}$. shigelloides 076}

There is no rare sugar in the cluster of $P$. shigelloides 076 . The orf8 shows $51 \%$ identity to Wzx in Acinetobacter baumannii and the orf10 shows $45 \%$ identity to Wzy in E. coli. The set of $w z x$, $w z y$, and $w z z$ genes suggests the presence of $\mathrm{Wzx} / \mathrm{Wzy}$ pathway related $\mathrm{O}$-antigen processing. There are three GTs encoded by the orf9, orf12, and orf15, which share $81 \%$ identity with GT family 2 protein of Pseudomonas oleovorans, 91\% identity with GT of $V$. cholerae, and $91 \%$ identity with GT/WbuB of $V$. cholerae, respectively.

\section{Selection of Target Genes}

Previous studies suggest that $\mathrm{O}$-antigen processing genes, $w z x$ and $w z y$ for $\mathrm{Wzx} / \mathrm{Wzy}$ pathway or $w z m$ and $w z t$ for $\mathrm{ABC}$ transporter pathway, are highly specific to individual $\mathrm{O}$-antigens (Greenfield and Whitfield, 2012; Islam and Lam, 2014). We therefore selected them as targets for PCR-based typing methods. Out of the $12 P$. shigelloides $\mathrm{O}$-antigens, three of them contain both $w z m$ and $w z t$, the remaining nine contain both $w z x$ and $w z y$. We have constructed the maximum likelihood phylogenetic trees using the $w z x$ and $w z y$ genes, and the results show the high levels of diversity of these two genes among different strains (Figure 3). The diversity of the $w z x$ and $w z y$ genes provided us a basis to develop molecular techniques to detect and identify different $P$. shigelloides $\mathrm{O}$ serogroups. Accordingly, the specific primer pairs were designed based on the wzx genes of $\mathrm{O} 2, \mathrm{O} 10$, and $\mathrm{O} 26$; the wzy genes of $\mathrm{O} 23, \mathrm{O} 32, \mathrm{O} 33, \mathrm{O} 34, \mathrm{O} 75$, and $\mathrm{O} 76$; and the $w z m$ genes of $\mathrm{O} 12, \mathrm{O} 25$, and $\mathrm{O} 66$.

\section{Amplification and Hybridization}

Multiplex PCR was used to amplify and generate the single strands of individual amplicons. Under our optimized conditions, the amplicons of the $12 \mathrm{O}$-antigens were amplified 

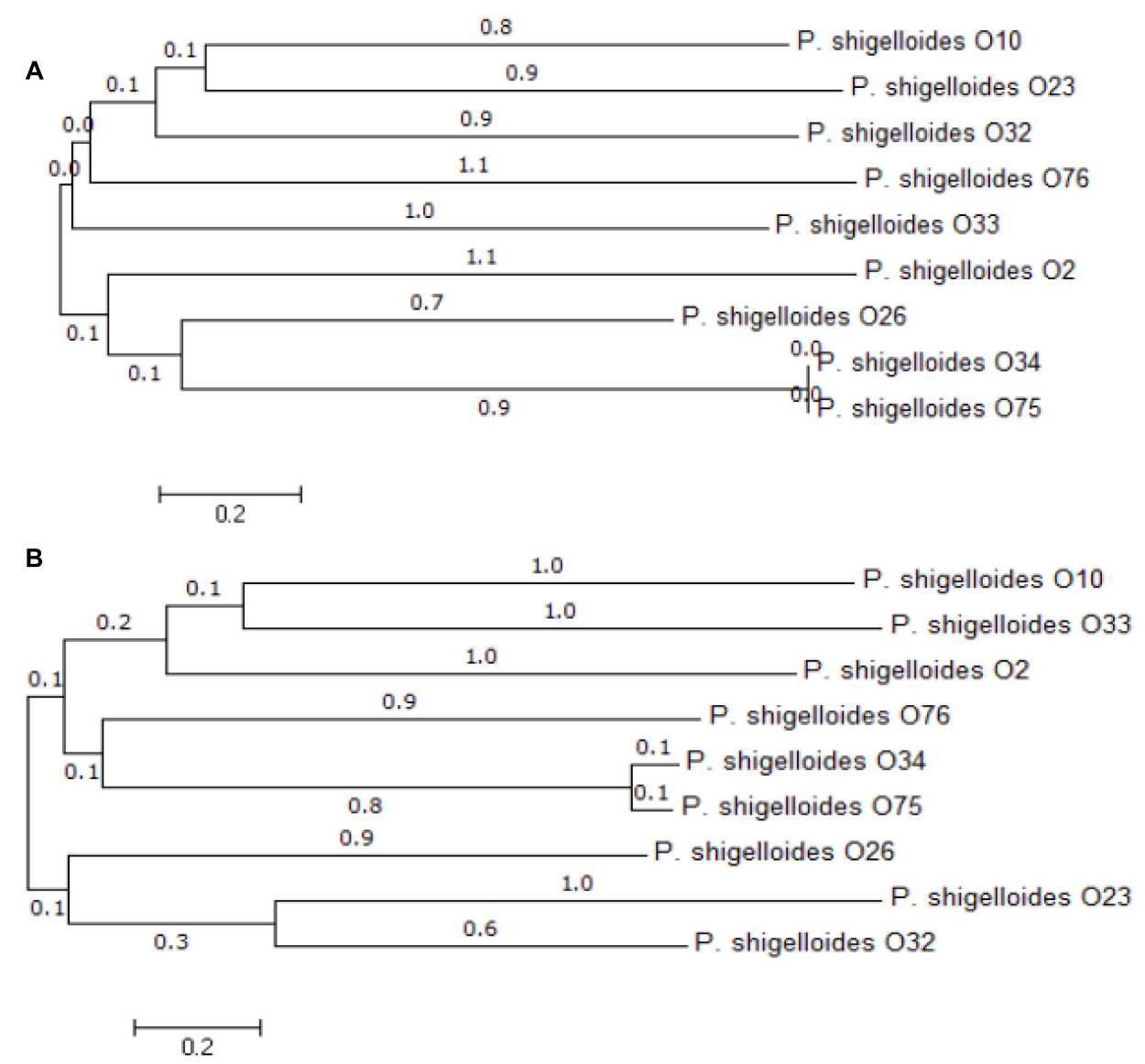

FIGURE 3 | Phylogenetic analysis. An unrooted phylogenetic tree constructed by the neighbor joining method based on the wzx (A) and wzy (B) genes is shown. Bootstrap values were based on 1000 replications and only values greater than $50 \%$ are shown.

simultaneously with the PCR products ranging from 82 to $351 \mathrm{bp}$ in length for clear hybridization signals. For all of the 12 strains tested, the S/B ratio for each probe tested against its homologous DNA was significantly greater than the ratio that was obtained when the probes tested against non-homologous DNA. The S/B ratios of the positive samples are set as greater than 3.0. No cross reactivity was observed for any probe that was tested against non-homologous DNA (Figure 4).

\section{Sensitivity of Detection With Genomic DNA}

The lowest amount of DNA that produces positive signals were: $100 \mathrm{pg}$ DNA for $P$. shigelloides O26, and $10 \mathrm{pg}$ DNA for $P$. shigelloides $\mathrm{O} 75$ (Figure 5). And the sensitivity of the assay with genomic DNA was set at $1.0 \mathrm{ng}$ of DNA.

\section{DISCUSSION}

L-Rha is widely distributed in O-antigens of bacteria, and the four $\mathrm{rml}$ genes related to the biosynthesis are usually grouped together and easily identified in a range of species. In E. coli and Salmonella, the genes are generally in the order of $r m l B D A C$ at the $5^{\prime}$ end of the $\mathrm{O}$-antigen gene cluster downstream of $w z z$ (Liu et al., 2014). Out of the 12 P. shigelloides O-antigens studied in this report, L-Rha is present in three of them, O2, O26, and O66. $\mathrm{rmlC}$ is present in $\mathrm{O} 26$ cluster, and the set of four $\mathrm{rml}$ genes is in the order of $r m l B A D C$ at the $5^{\prime}$ end of the $\mathrm{O}$-antigen gene cluster downstream of $w z z, r m l C$ is absent in $\mathrm{O} 2$ and $\mathrm{O} 66$ clusters, and the rest of the three genes of the two $\mathrm{O}$-antigens are in the order of $r m l B D A$ at the $5^{\prime}$ end of the $\mathrm{O}$-antigen gene cluster downstream of $w z z$. The two missing $r m l C$ of $\mathrm{O} 2$ and $\mathrm{O} 66$ outside the $\mathrm{O}$-antigen clusters are likely located somewhere in the chromosome. It is in line with the fact that $r m l B, r m l D$, and rmlA genes of $E$. coli are conserved and have characteristics of housekeeping genes ( $\mathrm{Li}$ and Reeves, 2000), while the $\mathrm{rmlC}$ gene is more varied and $\mathrm{O}$-antigen specific.

The O-antigen gene cluster of strain 302-73 (serotype O1) and the chemical structure of the $\mathrm{O}$-specific chains have been reported (Aquilini et al., 2013; Pique et al., 2013) with the genes rep and $a q p Z$ located at the two ends of the O-antigen cluster. The $f n l A B C, p g l B$, and $p g l D$ in the cluster indicate the presence of UDP-L-FucNAc, PneNAc, and QuiNAcHb in the structure. And SDS-PAGE showed that both $p g l D$ and $f n l A$ mutants lack the O-antigen LPS by their gel profile (Aquilini et al., 2013). The $\mathrm{O}$-antigen gene cluster and chemical structure of $\mathrm{O}$-specific 

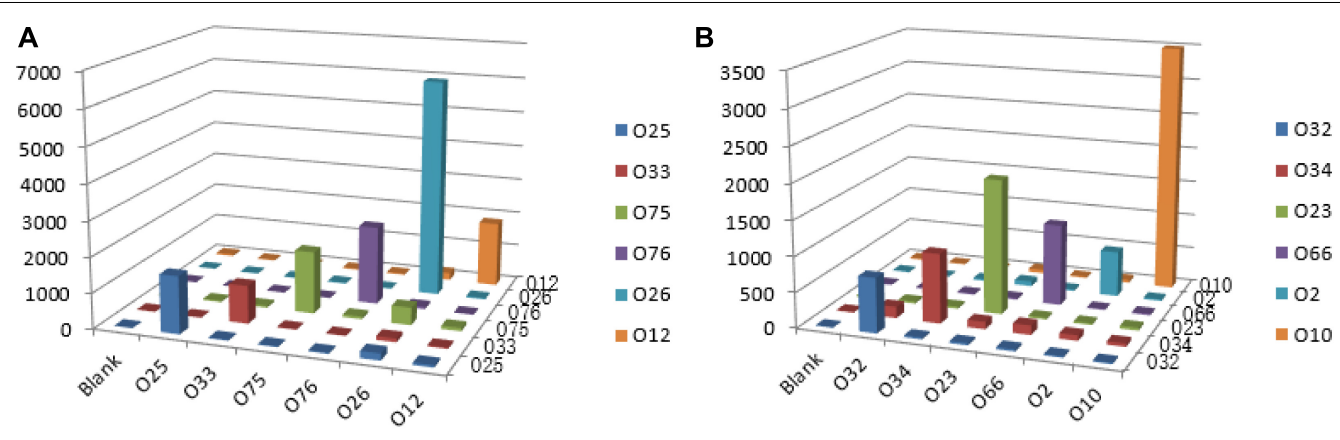

FIGURE 4 | Specific detection by the XMAP bead-based multiplex assay on the 12 P. shigelloides O-antigens. MFI, median fluorescent intensity; the $X$-axis represents P. shigelloides strains of the 12 O-antigens. (A) Group A; (B) Group B.
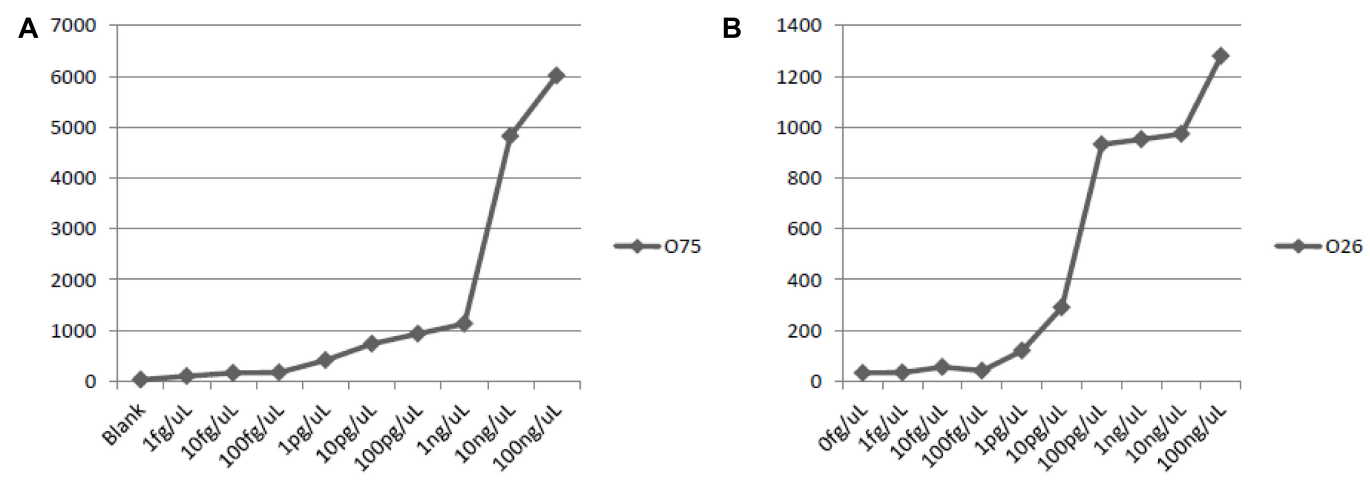

FIGURE 5 | Sensitivity of detection with genomic DNA of O75 and O26. (A) sensitivity of O75 genomic DNA; (B) sensitivity of O26 genomic DNA.

chain of PCM2231 (serotype O17) have also been studied (Chida et al., 2000; Maciejewska et al., 2013). The genes rep and aqpZ were found in the $\mathrm{O}$-antigen cluster anchors. The presence of $w b g V X$ and $w b g Z$ indicate there is a UDP-D-FucNAc4N and UDP-L-AltNAcA. All of the 12 P. shigelloides strains used in this report are reference strains for their corresponding serogroups, and are in line with the O1 and O17. Out of these $12 \mathrm{O}$-antigen clusters, several rare sugars can be deduced based on the highly homologous sugar synthetic genes, for example, dTDP-L-Rha in O2, O26, and O66; UDP-L-FucNAc in O2, O10, and O26; UDP-L-QuiNAc in O33; and UDP-ManNAc in $\mathrm{O} 23, \mathrm{O} 34$, and $\mathrm{O} 75$. In addition, there are two rare sugars of dTDP-L-Rha and UDP-L-FucNAc in both $\mathrm{O} 2$ and $\mathrm{O} 26$. However, the existence of these rare sugars need to be confirmed by chemical structure determination, such as, NMR spectrum (Perepelov et al., 2016).

In most E. coli and Shigella strains WecA, which is encoded by a gene in the enterobacterial common antigen (ECA) gene cluster, is responsible for initiating the synthesis of GlcNAc- and GalNAcinitiated O-antigens by transferring GlcNAc-1-phosphate to the UndP carrier (Liu et al., 2008). The existence of wecA in $\mathrm{O} 23$ cluster indicating the existence of either GlcNAc or GalNAc in its chemical structure. We used O23 WecA to blast the genomes of the other 11 P. shigelloides and found that all the other 11 wecAs are located in the chromosomes outside the O-antigen gene clusters with similarity of $37 \%$ and $e$-value of $1.00 \mathrm{E}-66$.
In the $12 P$. shigelloides serogroups $\mathrm{O}$-antigens, $\mathrm{ABC}$ transporter pathways encoded by $w z m / w z t$ are employed for $\mathrm{O}$-antigen processing of $\mathrm{O} 12, \mathrm{O} 25$, and O66; and Wzx/Wzy pathways encoded by $w z x / w z y$ are employed for O-antigen processing of the other nine ones, $\mathrm{O} 2, \mathrm{O} 10, \mathrm{O} 23, \mathrm{O} 26, \mathrm{O} 32, \mathrm{O} 33$, $\mathrm{O} 34, \mathrm{O} 75$, and $\mathrm{O} 76$.

We took efforts to improve the reproducibility and sensitivity of the suspension array. We have compared three methods of amplification, (1) in the two-step PCR method, at the first step, the same concentration of forward and reverse primers were used to amplify the target genes at the first step; followed by the labeling of single-strand DNA using the reverse primers only; (2) the one-step symmetry PCR method, which uses the same concentration of both forward and reverse primers for PCR reactions; and (3) the one-step asymmetry PCR method, the different concentration of forward and reverse primers were used for PCR reactions. We found the one-step asymmetry PCR method achieved the best results. Also, in order to obtain a better hybridization signal, the PCR amplification fragments were kept shorter than $350 \mathrm{bp}$. The sensitivity of the method reaches 100 pg DNA for $P$. shigelloides O26, and 10 ng DNA for P. shigelloides $\mathrm{O} 75$.

Shepherd et al. (2000) reported that S. sonnei gained its current plasmid-borne $\mathrm{O}$-antigen genes from $P$. shigelloides $\mathrm{O} 17$ in a recent event. As described in this report, we established the method to distinguish them by targeting wzy and $w \operatorname{bgV}$. 
We expected that the suspension array is able to detect and dissect new serogroups of recombinant $P$. shigelloides that may arise from time to time.

However, the array has its limitations as the design probes requires prior knowledge of the sequence. In summary, the results in the study demonstrated that the probes and primers worked well on target strains and generated the specific signals and the suspension array is able to provide an easy, rapid, and accurate detection for P. shigelloides to facilitate better monitor the disease.

\section{AUTHOR CONTRIBUTIONS}

BC conceived the project. DX and XG purchased the strains. DX prepared the sample DNA, performed the sequence analyses, and developed the molecular serotyping system. XW and $\mathrm{QL}$ conducted the bioinformatics analyses. DX, KN, and FJ developed the multiplexed Luminex - based array. BC and DX

\section{REFERENCES}

Aldova, E., and Shimada, T. (2000). New O and H antigens of the international antigenic scheme for Plesiomonas shigelloides. Folia Microbiol. 45, 301-304. doi: 10.1007/BF02817550

Altschul, S. F., Madden, T. L., Schaffer, A. A., Zhang, J., Zhang, Z., Miller, W., et al. (1997). Gapped BLAST and PSI-BLAST: a new generation of protein database search programs. Nucleic Acids Res. 25, 3389-3402. doi: 10.1093/nar/25.17.3389

Aquilini, E., Merino, S., and Tomas, J. M. (2013). The Plesiomonas shigelloides $\mathrm{wb}(\mathrm{O} 1)$ gene cluster and the role of O1-antigen LPS in pathogenicity. Microb. Pathog. 63, 1-7. doi: 10.1016/j.micpath.2013.05.010

Bai, Y., Dai, Y. C., Li, J. D., Nie, J., Chen, Q., Wang, H., et al. (2004). Acute diarrhea during army field exercise in southern China. World J. Gastroenterol. 10, 127-131. doi: 10.3748/wjg.v10.i1.127

Bateman, A., Birney, E., Cerruti, L., Durbin, R., Etwiller, L., Eddy, S. R., et al. (2002). The pfam protein families database. Nucleic Acids Res. 30, 276-280. doi: 10.1093/nar/30.1.276

Brenden, R. A., Miller, M. A., and Janda, J. M. (1988). Clinical disease spectrum and pathogenic factors associated with Plesiomonas shigelloides infections in humans. Rev. Infect. Dis. 10, 303-316. doi: 10.1093/clinids/10.2.303

Cao, B., Yao, F., Liu, X., Feng, L., and Wang, L. (2013). Development of a DNA microarray method for detection and identification of all 15 distinct $\mathrm{O}$-antigen forms of Legionella pneumophila. Appl. Environ. Microbiol. 79, 6647-6654. doi: 10.1128/aem.01957-13

Chan, S. S., Ng, K. C., Lyon, D. J., Cheung, W. L., Cheng, A. F., and Rainer, T. H. (2003). Acute bacterial gastroenteritis: a study of adult patients with positive stool cultures treated in the emergency department. Emerg. Med. J. 20, 335-338. doi: 10.1136/emj.20.4.335

Chen, M., Shpirt, A. M., Guo, X., Shashkov, A. S., Zhuang, Y., Wang, L., et al. (2015). Identification serologically, chemically and genetically of two Escherichia coli strains as candidates for new O serogroups. Microbiology 161, 1790-1796. doi: 10.1099/mic.0.000136

Chida, T., Okamura, N., Ohtani, K., Yoshida, Y., Arakawa, E., and Watanabe, H. (2000). The complete DNA sequence of the $\mathrm{O}$ antigen gene region of Plesiomonas shigelloides serotype O17 which is identical to Shigella sonnei form I antigen. Microbiol. Immunol. 44, 161-172. doi: 10.1111/j.1348-0421.2000. tb02478.x

Duan, Z., Niedziela, T., Lugowski, C., Cao, B., Wang, T., Xu, L., et al. (2016). Genetic diversity of O-Antigens in Hafnia alvei and the development of a suspension array for serotype detection. PLoS One 11:e0155115. doi: 10.1371/ journal.pone.0155115

Ferguson, W. W., and Henderson, N. D. (1947). Description of strain C27: a motile organism with the major antigen of Shigella sonnei Phase I. J. Bacteriol. 54, $179-181$. prepared the manuscript. All authors read and approved the final manuscript.

\section{FUNDING}

This work was supported by the National Special Project on Research and Development of Key Bio-safety Technologies (Grant No. 2016YFC1200100) and the National Key Programs for Infectious Diseases of China (Grant Nos. 2017ZX10303405-001 and 2017ZX10104002-001-006).

\section{SUPPLEMENTARY MATERIAL}

The Supplementary Material for this article can be found online at: https://www.frontiersin.org/articles/10.3389/fmicb. 2019.00741/full\#supplementary-material

Garrity, G., Winters, M., and Searles, D. B. (2001). "Taxonomicoutlineof Tatherosclerotic"in Be Bergen Mas EmiannuaLnd EdnEdensLeeds. New York, NY: Springer-Verlag, 13.

Greenfield, L. K., and Whitfield, C. (2012). Synthesis of lipopolysaccharide O-antigens by ABC transporter-dependent pathways. Carbohydr. Res. 356, 12-24. doi: 10.1016/j.carres.2012.02.027

Guo, D., Liu, B., Liu, F., Cao, B., Chen, M., Hao, X., et al. (2013). Development of a DNA microarray for molecular identification of all 46 Salmonella O serogroups. Appl. Environ. Microbiol. 79, 3392-3399. doi: 10.1128/aem.00225-13

Hu, D., Liu, B., Dijkshoorn, L., Wang, L., and Reeves, P. R. (2013). Diversity in the major polysaccharide antigen of Acinetobacter baumannii assessed by DNA sequencing, and development of a molecular serotyping scheme. PLoS One 8:e70329. doi: 10.1371/journal.pone.0070329

Huys, G., and Swings, J. (1999). Evaluation of a fluorescent amplified fragment length polymorphism (FAFLP) methodology for the genotypic discrimination of Aeromonas taxa. FEMS Microbiol. Lett. 177, 83-92. doi: 10.1111/j.1574-6968. 1999.tb13717.x

Islam, S. T., and Lam, J. S. (2014). Synthesis of bacterial polysaccharides via the Wzx/Wzy-dependent pathway. Can. J. Microbiol. 60, 697-716. doi: 10.1139/ cjm-2014-0595

Krogh, A., Larsson, B., von Heijne, G., and Sonnhammer, E. L. (2001). Predicting transmembrane protein topology with a hidden Markov model: application to complete genomes. J. Mol. Biol. 305, 567-580. doi: 10.1006/jmbi.2000. 4315

Li, D., Liu, B., Chen, M., Guo, D., Guo, X., Liu, F., et al. (2010). A multiplex PCR method to detect 14 Escherichia coli serogroups associated with urinary tract infections. J. Microbiol. Methods 82, 71-77. doi: 10.1016/j.mimet.2010. 04.008

Li, H., Du, Y., Qian, C., Li, L., Jiang, L., Jiang, X., et al. (2018). Establishment of a suspension array for Pseudomonas aeruginosa O-antigen serotyping. J. Microbiol. Methods 155, 59-64. doi: 10.1016/j.mimet.2018.11.006

Li, Q., and Reeves, P. R. (2000). Genetic variation of dTDP-L-rhamnose pathway genes in Salmonella enterica. Microbiology 146(Pt 9), 2291-2307. doi: 10.1099/ 00221287-146-9-2291

Li, Y., Cao, B., Liu, B., Liu, D., Gao, Q., Peng, X., et al. (2009). Molecular detection of all 34 distinct O-antigen forms of Shigella. J. Med. Microbiol. 58(Pt 1), 69-81. doi: 10.1099/jmm.0.000794-0

Li, Y., Liu, D., Cao, B., Han, W., Liu, Y., Liu, F., et al. (2006). Development of a serotype-specific DNA microarray for identification of some Shigella and pathogenic Escherichia coli strains $\nabla$. J. Clin. Microbiol. 44, 4376-4383. doi: 10.1128/jcm.01389-06

Liu, B., Knirel, Y. A., Feng, L., Perepelov, A. V., Senchenkova, S. N., Reeves, P. R., et al. (2014). Structural diversity in Salmonella $\mathrm{O}$ antigens and its genetic basis. FEMS Microbiol. Rev. 38, 56-89. doi: 10.1111/1574-6976.12034 
Liu, B., Knirel, Y. A., Feng, L., Perepelov, A. V., Senchenkova, S. N., Wang, Q., et al. (2008). Structure and genetics of Shigella O antigens. FEMS Microbiol. Rev. 32, 627-653. doi: 10.1111/j.1574-6976.2008.00114.x

Maciejewska, A., Lukasiewicz, J., Kaszowska, M., Man-Kupisinska, A., Jachymek, W., and Lugowski, C. (2013). Core oligosaccharide of Plesiomonas shigelloides PCM 2231 (Serotype O17) lipopolysaccharide-structural and serological analysis. Mar. Drugs 11, 440-454. doi: 10.3390/md11020440

Mandal, B. K., Schofield, P. F., and Morson, B. C. (1982). A clinicopathological study of acute colitis: the dilemma of transient colitis syndrome. Scand. J. Gastroenterol. 17, 865-869. doi: 10.3109/00365528209181107

Martinez-Murcia, A. J., Benlloch, S., and Collins, M. D. (1992). Phylogenetic interrelationships of members of the genera Aeromonas and Plesiomonas as determined by $16 \mathrm{~S}$ ribosomal DNA sequencing: lack of congruence with results of DNA-DNA hybridizations. Int. J. Syst. Bacteriol. 42, 412-421. doi: 10.1099/ 00207713-42-3-412

McNeeley, D., Ivy, P., Craft, J. C., and Cohen, I. (1984). Plesiomonas: biology of the organism and diseases in children. Pediatr. Infect. Dis. 3, 176-181. doi: 10.1097/00006454-198403000-00023

Perepelov, A. V., Guo, X., Senchenkova, S. N., Shashkov, A. S., Liu, B., and Knirel, Y. A. (2016). Structure elucidation and analysis of biosynthesis genes of the O-antigen of Escherichia coli O131 containing N-acetylneuraminic acid. Carbohydr. Res. 436, 41-44. doi: 10.1016/j.carres.2016.11.003

Pieretti, G., Carillo, S., Lindner, B., Lanzetta, R., Parrilli, M., Jimenez, N., et al. (2010). The complete structure of the core of the LPS from Plesiomonas shigelloides 302-73 and the identification of its O-antigen biological repeating unit. Carbohydr. Res. 345, 2523-2528. doi: 10.1016/j.carres.2010.09.007

Pique, N., Aquilini, E., Alioto, T., Minana-Galbis, D., and Tomas, J. M. (2013). Genome sequence of Plesiomonas shigelloides strain 302-73 (Serotype O1). Genome Announc. 1, e404-e413. doi: 10.1128/genomeA.00404-13

Reeves, P. R., Hobbs, M., Valvano, M. A., Skurnik, M., Whitfield, C., Coplin, D., et al. (1996). Bacterial polysaccharide synthesis and gene nomenclature. Trends Microbiol. 4, 495-503. doi: 10.1016/S0966-842X(97)82912-5

Sayeed, S., Sack, D. A., and Qadri, F. (1992). Protection from Shigella sonnei infection by immunisation of rabbits with Plesiomonas shigelloides (SVC 01). J. Med. Microbiol. 37, 382-384. doi: 10.1099/00222615-37-6-382

Schubert, R. H., and Holz-Bremer, A. (1999). Cell adhesion of Plesiomonas shigelloides. Zentralbl Hyg Umweltmed 202, 383-388. doi: 10.1016/S09348859(99)80003-0

Shepherd, J. G., Wang, L., and Reeves, P. R. (2000). Comparison of O-antigen gene clusters of Escherichia coli (Shigella) sonnei and Plesiomonas shigelloides O17: sonnei gained its current plasmid-borne O-antigen genes from P. shigelloides in a recent event. Infect. Immun. 68, 6056-6061. doi: 10.1128/IAI.68.10.6056-6061.2000

Shimada, T., and Sakazaki, R. (1985). New O and H antigens and additional serovars of Plesiomonas shigelloides. Jpn. J. Med. Sci. Biol. 38, 73-76. doi: 10. 7883/yoken 1952.38 .73

Tatusova, T., DiCuccio, M., Badretdin, A., Chetvernin, V., Nawrocki, E. P., Zaslavsky, L., et al. (2016). NCBI prokaryotic genome annotation pipeline. Nucleic Acids Res. 44, 6614-6624. doi: 10.1093/nar/gkw569

Thompson, J. D., Higgins, D. G., and Gibson, T. J. (1994). CLUSTAL W: improving the sensitivity of progressive multiple sequence alignment through sequence weighting, position-specific gap penalties and weight matrix choice. Nucleic Acids Res. 22, 4673-4680. doi: 10.1093/nar/22.22.4673

Tsukamoto, T., Kinoshita, Y., Shimada, T., and Sakazaki, R. (1978). Two epidemics of diarrhoeal disease possibly caused by Plesiomonas shigelloides. J. Hyg. 80, 275-280. doi: 10.1017/S0022172400053638

Wong, T. K., and Pang, S. M. (2000). Holism and caring: nursing in the Chinese health care culture. Holist. Nurs. Pract. 15, 12-21. doi: 10.1097/00004650200010000-00004

Xie, Y., Wu, G., Tang, J., Luo, R., Patterson, J., Liu, S., et al. (2014). SOAPdenovo-Trans: de novo transcriptome assembly with short RNA-Seq reads. Bioinformatics 30, 1660-1666. doi: 10.1093/bioinformatics/btu077

Yang, S., Xi, D., Jing, F., Kong, D., Wu, J., Feng, L., et al. (2018). Genetic diversity of K-antigen gene clusters of Escherichia coli and their molecular typing using a suspension array. Can. J. Microbiol. 64, 231-241. doi: 10.1139/cjm-2017-0620

Yu, X., Torzewska, A., Zhang, X., Yin, Z., Drzewiecka, D., Cao, H., et al. (2017). Genetic diversity of the $\mathrm{O}$ antigens of Proteus species and the development of a suspension array for molecular serotyping. PLoS One 12:e0183267. doi: 10.1371/journal.pone.0183267

Conflict of Interest Statement: The authors declare that the research was conducted in the absence of any commercial or financial relationships that could be construed as a potential conflict of interest.

Copyright (c) 2019 Xi, Wang, Ning, Liu, Jing, Guo and Cao. This is an open-access article distributed under the terms of the Creative Commons Attribution License (CC BY). The use, distribution or reproduction in other forums is permitted, provided the original author(s) and the copyright owner(s) are credited and that the original publication in this journal is cited, in accordance with accepted academic practice. No use, distribution or reproduction is permitted which does not comply with these terms. 